Received: 2014.08.03

Accepted: 2014.08.28

Published: 2015.01 .07
Authors' Contribution:

Study Design A

Data Collection B

Statistical Analysis C

Data Interpretation D

Manuscript Preparation E

Literature Search F

Funds Collection G

\title{
Atrial Fibrillation Electrical Remodelling via Ablation of the Epicardial Neural Networks and Suprathreshold Stimulation of Vagosympathetic Nerve
}

\author{
ABC Yanmei Lu* \\ AF Juan Sun* \\ CD Xianhui Zhou \\ B Qinquan Sun \\ BC Shuai Sun \\ ADFG Baopeng Tang
}

Department of Cardiology, First Affiliated Hospital, Xinjiang Medical University, Urumqi, Xinjiang, China
Corresponding Author: Source of support:

* These authors contributed equally to this work and should be considered co-first authors Baopeng Tang, e-mail: baopengtang@126.com

This work was supported by the National Natural Science Foundation of China (NSFC81260037), Science Foundation of First Affiliated Hospital, Xinjiang Medical University (2012YFY23)

Background:

Material/Methods:

Results:

Conclusions:

MeSH Keywords:

Full-text PDF:
Numerous studies have shown that the cardiac autonomic nervous system (CANS) is involved in the occurrence and persistence of atrial fibrillation (AF). The CANS is commonly considered to consist of the extrinsic and intrinsic autonomic nerves. The influence of exogenous and endogenous nerve stimulation plexus ablation on pulmonary vein sleeves and atrial myocardium provides important information in understanding the occurrence and persistence of AF. Vagosympathetic nerve stimulation and epicardial neural networks are important participants in atrial electrical remodelling (AER). Elucidation of the changes in the electrophysiological indicators of the atrial and pulmonary veins caused by epicardial neural network ablation and autonomic nerve stimulation may provide a theoretical basis for the clinical treatment of AF.

A total of 13 beagle dogs were randomly divided into 2 groups: the control group $(n=6)$, which was treated with a simple rapid atrial pacing (RAP) for $6 \mathrm{~h}$, and the experimental group $(n=7)$, which was treated with RAP+vagus nerve stimulation (VNS) for 6 h. Both groups were treated with epicardial ganglia plexus (GP) ablation after 6 h. We measured the monophasic action potential (MAP), various parts of the effective refractory period (ERP), and $A F$ induction rate before and after pacing or ablation.

With the extension of the pacing time, the atrial MAP and ERP of the 2 groups shortened and returned to normal after ablation plexus. After GP ablation, the atrial AF-induced rate did not decrease significantly compared with that of the pulmonary vein.

Vagus nerve threshold stimulation exacerbated the deterioration of electrical remodelling, whereas the epicardial neural network ablation blocked or reversed the AER.

\section{Atrial Fibrillation - Autonomic Nervous System • Catheter Ablation}

http://www.medscimonit.com/abstract/index/idArt/892156

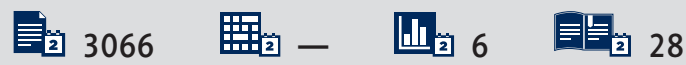




\section{Background}

Atrial fibrillation (AF) is one of the major cardiovascular disorders seriously affecting human health and quality of life. The incidence of AF gradually increases along with the age of the population. Understanding the pathogenesis of AF can help provide more effective clinical treatment options [1]. Numerous studies have shown that the cardiac autonomic nervous system (CANS) is involved in the occurrence and persistence of AF. CANS is commonly considered to consist of the extrinsic and intrinsic autonomic nerves. The external cardiac autonomic nervous system (ECANS) consists of the brain nuclei, vagus nerve, paravertebral ganglia chain, and the postganglionic neurons axons distributed next to the heart. The intrinsic cardiac autonomic nervous system (ICANS) is composed of several ganglia plexus and interconnected nerve or neurons [2-5]. The influence of the exogenous and endogenous nerve stimulation plexus ablation on pulmonary vein sleeves and atrial myocardium provides important information needed to understand the occurrence and persistence of AF [6-9]. However, detailed studies on the occurrence and maintenance of AF are lacking.

Paroxysmal AF has been clinically proven to occur in a population with normal cardiac anatomy structures and electrical remodelling (AER). AER is the theoretical basis of "AF begets $A F "[10,11]$. An experimental animal model was constructed using AER to study the physiological changes in the electrical remodelling indicators during atrial myocardium electrical remodelling. The vagus nerve of the extrinsic autonomic nervous system was stimulated to understand the effect of the nerve on the atrial myocardium. The ERP of the various parts of the pulmonary veins and electrophysiological index changes the induction of AF during the AER period, which further confirms the influence of the ECANS on the heart and demonstrates the significance of the clinical treatment.

\section{Material and methods}

\section{Animal preparation}

A total of 13 healthy adult beagles, males and females, with weight of $12.0 \pm 1.5 \mathrm{~kg}$ and age of 1 year to 2 years, were provided by the First Affiliated Hospital of Xinjiang Medical University Animal Center. Approximately $30 \mathrm{mg} / \mathrm{kg}$ sodium pentobarbital was given for anaesthesia. All dogs were given a ventilator in a room with air circulation that had positive pressure and an insulation blanket to maintain the body temperature at $36.5 \pm 1.5^{\circ} \mathrm{C}$. We opened the chest at the fourth rib from the left and right sides. Subsequently, a multi-electrode was sewn over and below the pulmonary vein of the left and right sides, the left and right atrium and the left atrial appendage and dextra for MAP pacing and recording (Figure 1A, 1B). The canine vagus nerve on the right side of the neck was cut. The parallel needles were then inserted to stimulate the vagus nerve to produce effects. To avoid nerve drying, liquid paraffin infiltration was used. The connected ECG recording device, connecting leads, and basic vital signs, such as oxygen saturation and arterial blood pressure, were monitored. This study was carried out in strict accordance with the recommendations in the Guide for the Care and Use of Laboratory Animals of the National Institutes of Health. The animal use protocol was reviewed and approved by the Institutional Animal Care and Use Committee (IACUC) of Xinjiang Medical University.

\section{Canine acute AF model}

The left atrial appendage (LAA) was sewn with electrodes with pacing frequency of 1200 times/min and pacing voltage that was 2 times that of the pacing threshold (TH). The auricula dextra and the left and right pulmonary vein sleeves were sewn with electrodes for the pacing and recording of ERP. The stimulation and recording of all the program aspects were measured using the electrodes of the multi-conductive polygraph (Lead-7000 EP Control, Sichuan Jinjiang, China), which were connected to the heart. The pacing was suspended when ERP was measured by decreasing S1S2 (S1-S1=300 ms, S1:S2=8:1, V=2×TH). The AF-induced rate was stimulated using S1S1 for $30 \mathrm{~s}$ and was stopped to observe whether AF could be maintained for $5 \mathrm{~s}$ or more. A positive result was defined as a successful one-time induction. Each site measurement was repeated 3 times. AF was defined as having more than $5 \mathrm{~s}$ of irregular 500 beats/ min heart rate with an irregular atrioventricular conduction.

The anterior walls of the left and right atrium anterior were sewn with a multi-electrode for MAP recording under different conditions (Figure 2) and the multi-electrode was connected to a conductive physiological cardiac multi-meter (Lead-7000 EP amp, Sichuan Jinjiang, China). MAP is the average of the extracellular action potentials of a cell group and is an important bridge that combines the physiological changes in cardiac cell electrophysiology and clinical electrophysiology. The use of MAP changes for reflecting the atrial myocardium electrical remodelling process can provide important information for clinical treatment. These changes also reflect early changes in the electrophysiological properties of the atrial myocardium [12]. A 90\% repolarization of action potential duration (APD90) based on MAP was used for data comparison

\section{Electrical nerve stimulation}

The left vagus sympathetic trunk is commonly used to treat noncardiovascular system disorders because it was thought to produce cardiovascular effects [13]. This research produced a major stimulus to the right of the vagus nerve for cardiovascular effects. A pair of Teflon-coated silver wires (0.1-mm diameter) 

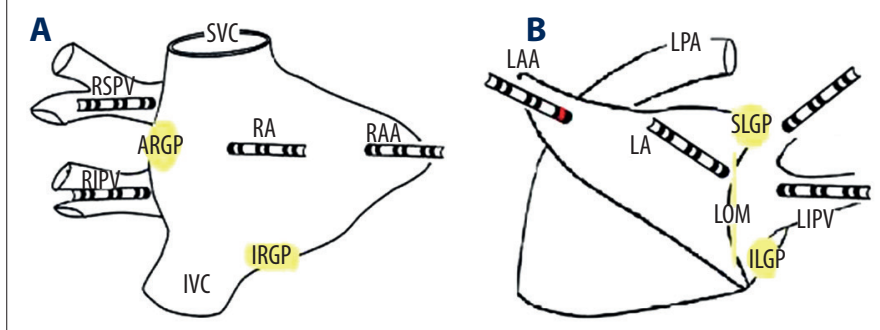

C

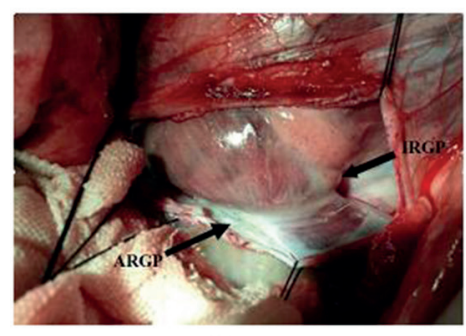

D

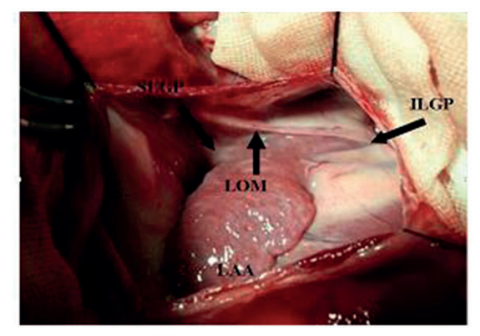

A
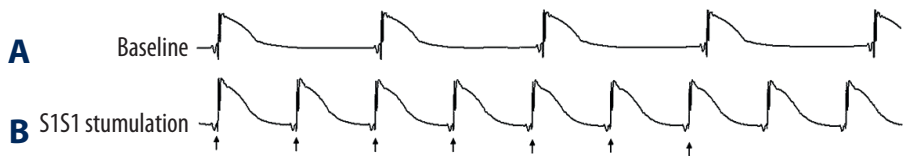

C s152 stumulation

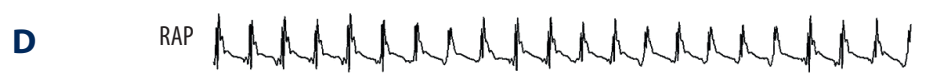

E

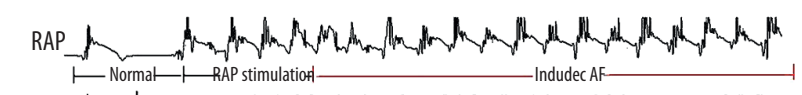

F

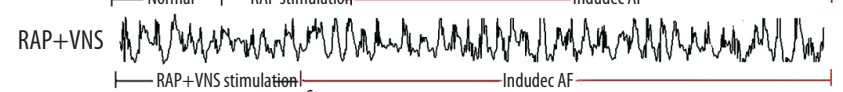

Figure 1. (A-D) Experimental model diagram of multi-electrode placed in the heart surface. Yellow was for the positioning plexus anatomy, red for RAP, the pacing frequency of left atrial was 1200 bpm; SVC=superior vena cava; IVC=inferior vena cava; $\mathrm{RA}=$ right atrium; $\mathrm{RAA}=$ right atrial appendage; $R S P V=$ right superior pulmonary vein; RIPV= right inferior pulmonary vein; $A R G P=$ anterior right ganglionated plexi; IRGP=inferior right ganglionated plexi; LA=left atrium; $L A A=$ left atrial appendage; $L S P V=$ left superior pulmonary vein; LIPV=left inferior pulmonary vein; LPA=left pulmonary artery; SLGP=superior left ganglionated plexi; ILGP=inferior left ganglionated plexi; LOM=ligament of Marshall.

Figure 2. Atrial MAP record. (A) Indicates atrial MAP recording the normal state. (B) Denotes MAP stimulated by the S1S1 program. (C) Represents MAP stimulated by S1S2 program. (D) Means MAP of RAP group. (E) Represents induced atrial fibrillation record of RAP group. (F) Indicates MAP record in RAP+VNS-induced atrial fibrillation group. $\uparrow$ was for the S1 stimulus, $\uparrow$ was for S2 stimulus. The figure shows that significant changes in morphology of MAP and morphological disorders of MAP in AF were induced. was inserted parallel to the sympathetic vagus trunk with a pitch of $2 \mathrm{~mm}$ to $3 \mathrm{~mm}$, ensuring that the silver-plated needle did not penetrate the neural stem. Vagal nerve stimulation (VNS) was conducted by stimulating the vagus nerve stem with a Grass stimulator (S88X, Astro-Med Inc., Warwick, RI, USA) using high-frequency electrical stimulation (high-frequency stimulation, HFS; $20 \mathrm{~Hz}$, interval $0.1 \mathrm{~ms}$, square wave) to delay the VNS minimum voltage of the sinus rhythm (as defined by TH). The voltage above that of the threshold was selected to cause the sinus rate to decrease by $50 \%$ or to promote a second-degree atrioventricular block. This voltage was regarded as the stimulation voltage. The required voltage ranged from $2.0 \mathrm{~V}$ to $3.5 \mathrm{~V}$.

\section{GP ablation}

Handheld 2-way electrodes were used to provide pressure on the plexus surface for stimulation until the occurrence of the vagus effect (HFS; $20 \mathrm{~Hz}, 0.5 \mathrm{~V}$ to $12 \mathrm{~V}$ and pulse width $1 \mathrm{~ms}$ to $10 \mathrm{~ms}$ ). The plexus positioning supported the following 5 main distribution areas of the plexus on the basis of a previous report (Figure 1C, 1D): (1) anterior right ganglionated plexi (ARGP) located between the upper and lower pulmonary vein, near the right pulmonary vein; (2) inferior right ganglionated plexi (IRGP) located in the junction of the inferior vena cava and atrium cordis; (3) superior left ganglionated plexi (SLGP) located between the left upper pulmonary vein and left pulmonary artery; (4) inferior left ganglionated plexi (ILGP) located at the junction of the posterior wall of the left lower pulmonary vein and left atrium; and (5) another part of the plexus nerve located within the ligament of Marshall (LOM) [14]. Radiofrequency ablation (RF) was conducted. Positioning GP ablation was then performed $(460 \mathrm{kHz},<32.5 \mathrm{~W}, \mathrm{HL}-75 \mathrm{~F}$, Sichuan Jinjiang, China). The presence of complete plexus ablation (ARGP+IRGP+SLGP+ILGP+LOM) indicated that all positive 

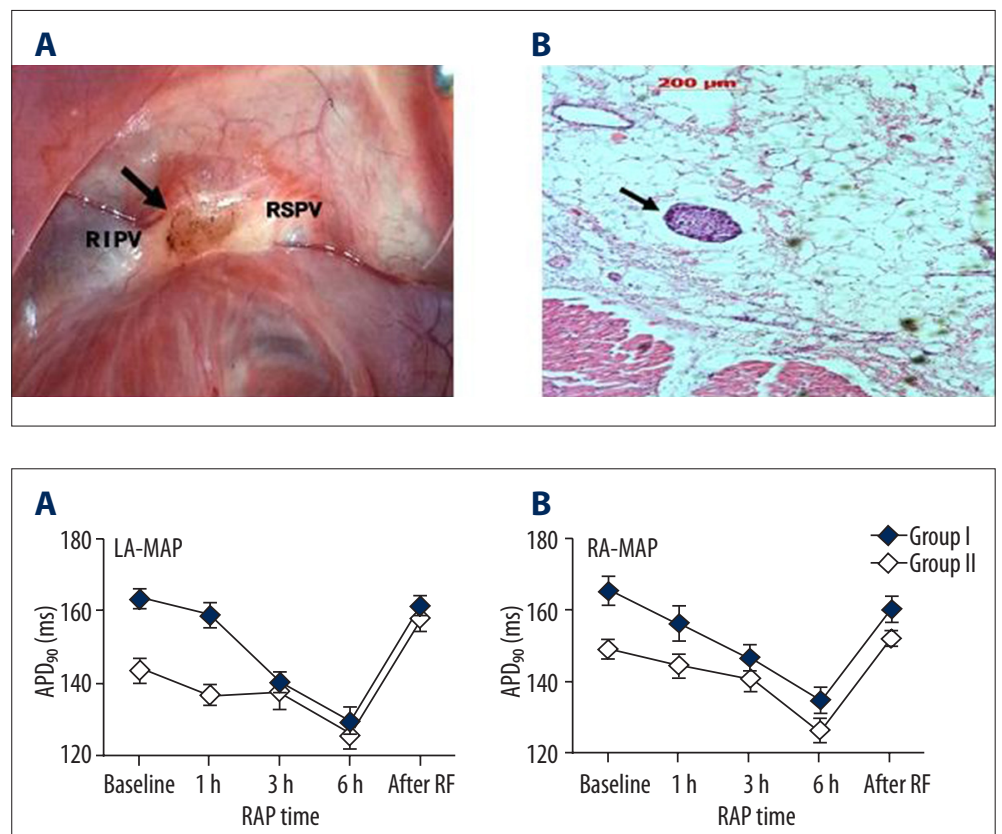

Figure 3. ARGP plexus ablation. (A) Shows surface tissue (yellow) after ARGP ablation. All positive reactions disappeared under strongest voltage stimulation. (B) Shows GP plexus degeneration after HE staining, $\rightarrow$ indicates GP.
Figure 4. APD90 trend chart in 2 atrial myocardia. (A) Show the left atrium MAP and (B) shows the right atrial MAP, $P<0.05$. reactions disappeared after stimulation using the strongest voltage (12 V). GP tissue was extracted after the end of the HE staining experiment to verify the success of the ablation (Figure 3 ).

\section{Experimental route}

Group $1(n=6)$ : rapid left atrial appendage pacing (RAP) at 1200 times/min was administered for 6 h. Subsequently, GP (ARGP+IRGP+SLGP+ILGP+LOM) ablation was performed.

Group $2(n=7)$ : RAP was combined with neck VNS for $6 \mathrm{~h}$ after performing GP (ARGP+IRGP+SLGP+ILGP+LOM) ablation.

The ERP and AF induction rate of RSPV, RIPV, RAA, LSPV, LIPV and LAA in $2 \times$ TH based on ablation times of 1,3 and $6 \mathrm{~h}$, as well as the MAP of RA and LA, were measured in both groups. The base value of Group 1 did not undergo any interventions. By comparison, the base value of Group 2 was taken from the value of the stimulation of only the vagus nerve stem.

\section{Statistical analysis}

Measurement results are presented as mean \pm standard deviation. The induction rate for APD90, ERP, and AF at each time point was analyzed using ANOVA. The APD90 and ERP data before and after ablation were compared using the t-test, and the $A F$ induction rates before and after ablation were compared using the chi-square test. Statistical analysis was processed using the SPSS17.0 statistical package. A value of $P<0.05$ was considered statistically significant, and a value of $P<0.01$ indicated a significant difference.

\section{Results}

Throughout the experiment, the blood pressure of the animals remained stable without any sign of heart failure caused by rapid pacing. The vagus nerve stem was kept moist without discoloration and changes from the stimulating effect, thereby confirming that the neural stem was uninjured.

\section{Changes in the atrial MAP}

The left and right atrial MAP tests showed that the MAP morphology changes under different conditions (Figure 2). With the extension of the stimulus time, APD90 had varying degrees of shortening in both groups. The base values of Group 1 and Group 2 were $163.4 \pm 2.97 \mathrm{~ms}$ and $143.8 \pm 3.19 \mathrm{~ms}$, respectively. APD90 began to shorten $1 \mathrm{~h}$ after stimulation and was reduced to the lowest value (RAP group $129.8 \pm 3.7 \mathrm{~ms}$, RAP+VNS group $125.8 \pm 3.96 \mathrm{~ms}$, $P<0.05)$ after $6 \mathrm{~h}$. After performing GP ablation, the atrial APD90 recovered to its pre-stimulus levels $(P<0.05)$. However, the APD90 of the left and right atria had the most obvious downward trend upon stimulation of the walking nerve (Figure 4).

\section{ERP changes of the pulmonary vein and the left and right atrial appendage}

The base ERP value of Group 2 was shorter than that of Group $1(P<0.05)$. ERP in the different parts of the atria rapidly shortened within $1 \mathrm{~h}(\mathrm{P}<0.05)$, which was followed by a decrease in the shortening rate to the lowest value after $6 \mathrm{~h}(\mathrm{P}<0.01)$. After GP ablation, the ERP of the different parts of the atria were restored to the levels measured prior to the intervention (Figure 5). 

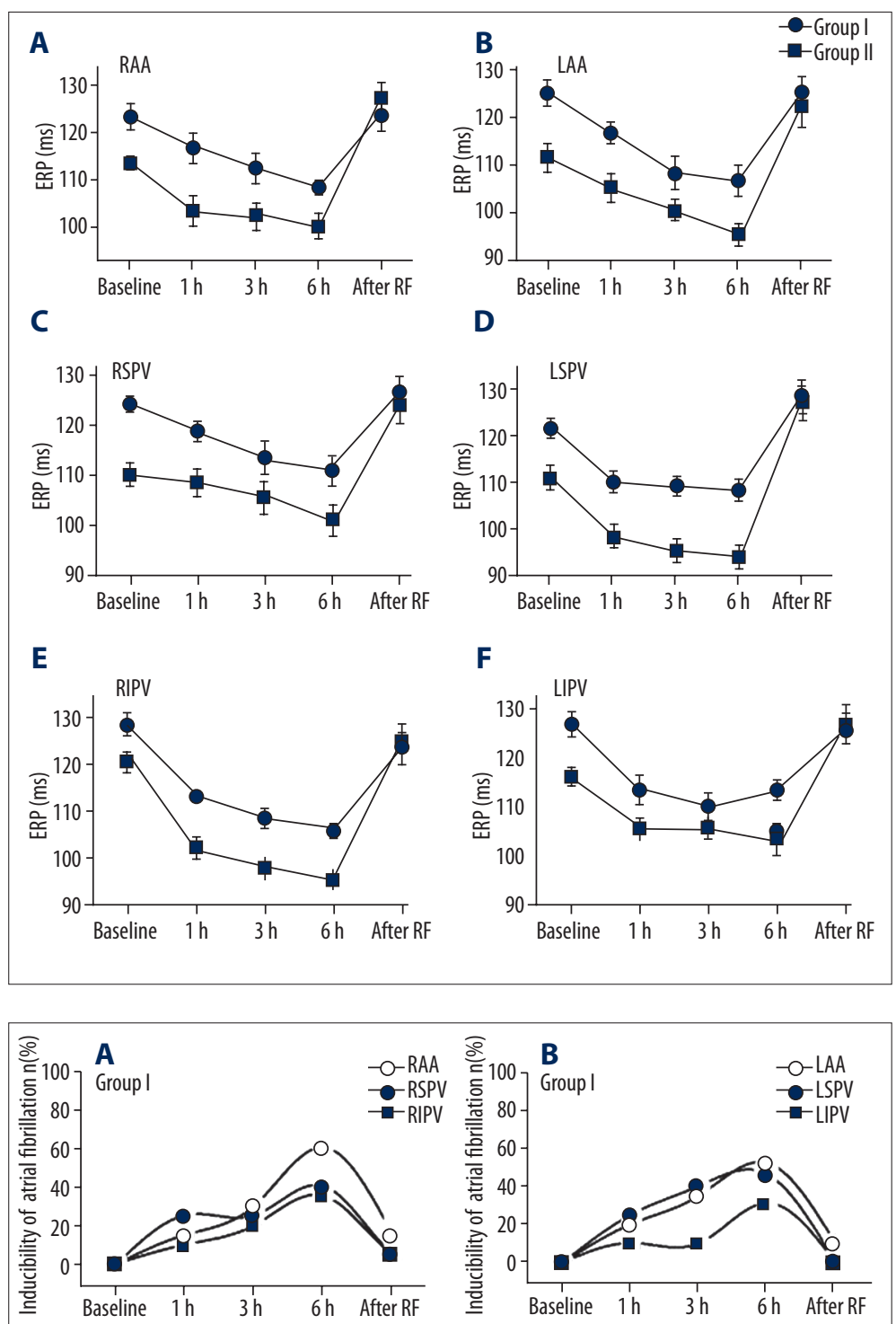

C
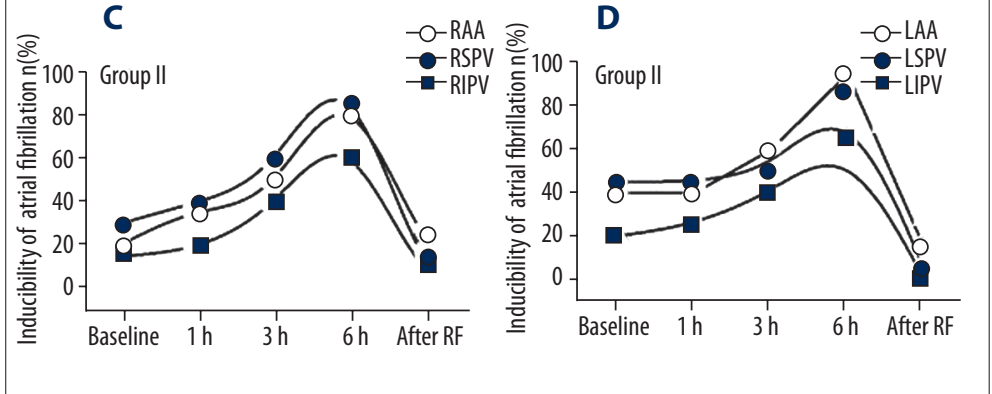

Figure 5. (A-F) ERP trend chart of various parts.

Figure 6. (A-D) Comparisons of the atrial fibrillation induced rate.

\section{Comparison of the left and right pulmonary vein and atria and the right $A F$ induction rate}

The AF induction rate in the different parts increased significantly with the extension of the intervention time. The induction rate of Group 2 at each time point was higher than that of Group $1(\mathrm{P}<0.05)$. The AF induction rates in both groups reached their highest values at $6 \mathrm{~h}$, with the left atrial appendage in group 2 reaching $100 \%$. After GP ablation, the induced rates by the pulmonary vein atrial stimulation of the 2 groups were significantly reduced $(P<0.05)$, although the left and right $\mathrm{AF}$ induction rate was lower after ablation. However, the induced AF was still detectable (Figure 6). 


\section{Discussion}

\section{Major findings}

APD90, which appeared in the atrial MAP of both groups, was shortened after GP ablation, but recovered to a level near that of the pre-intervention level. The ERP base values of all parts in Group 2 were shorter compared with that of Group 1 and were gradually shortened with the extension of the stimulus time, which was consistent with the MAP and ERP in both groups after GP ablation restoration to that of the pre-intervention level. The pacing of the AF was more easily induced by vagus nerve stem stimulation than by simple pacing. The atrial-induced AF rate after GP ablation decreased significantly compared with that of the pulmonary vein, suggesting that exogenous nerve stimulation increased atrial electrical remodelling. In addition, this finding suggests that epicardial ablation of the neural network can temporarily reverse the effects of nerve input by electrical remodelling of AF; that is, interference with the endogenous autonomic nerve could block the exogenous autonomic nerves. However, the effects of eliminating the exogenous nerve on the induced rate after GP ablation were not as significant as the effect of the pulmonary vein.

\section{AF begets AF}

Since the $20^{\text {th }}$ century, AF has been studied in various animal models developed for clinical research. Dogs have been the most popular species for use in such models because the hearts of dogs deliver an ion channel distribution and channel activity similar to those of humans, and the electrocardiogram and conduction time are also quite similar to those in humans; therefore, dogs are considered the best species for use in preclinical study of electrical activities of human hearts. Morillo et al. successfully constructed a model for right atrial appendage pacing [15]. In another study, Wijffels proposed the "AF begets AF" concept based on RAP $[16,17]$. A successful model for a drug-induced damaged valve in surgery or an induced AF by stimulating nerves should be based on the influence of AER on the atria. Nattel et al. further clarified that the AER was mainly transient for the outward potassium currents, reducing L-type calcium current, cell ultrastructure abnormalities, and abnormal calcium handling [18]. This phenomenon subsequently leads to physiological indexes, such as shortened ERP, increased ERPD, and increased AF-induced rate [19]. The AF model was successfully constructed in this work, and changes in the electrophysiological parameter were found to be in line with previous reports. The ERP of all parts gradually shortened with the extension of time, and the AFinduced rate significantly increased. We also confirmed that the MAP change was significant and that the changes in MAP are reversible, which proves that electrical remodelling is the ion flow of each change involved in the action potential. Therefore, the action potentials, as well as the ERP and other indexes, appeared to be abnormalities. The cycle of electrical remodelling can explain why patients had frequent episodes of paroxysmal AF in clinical studies. Paroxysmal AF eventually translated into persistent or even permanent AF; thus, "AF begets AF".

\section{Vagus nerve stimulation and GP ablation}

This study showed that activation of the CANS has an important role in the induction and persistence of AF. Exogenous vagus nerve stimulation can shorten ERP, reduce atrial reentrant length of the wave and is more prone to $\operatorname{AF}[20,21]$.

The comparison of data from RAP and RAP+VNS experiment groups revealed the following results: (1) the two 2 initial base values had significant differences; (2) atrial APD90 gradually shortened with time under vagus-mediated condition; (3) shortening of all ERP parts was enhanced; and (4) the induced rate of AF increased, but the electrophysiological indicators in various parts after GP ablation recovered to nearly the base values without vagal stimulation. Previous studies confirmed that either RAP or VNS provided GP with high activity because of the activation of endogenous neurons in to varying degrees. Thus, GP was in a highly active state, resulting in atrial electrical remodelling. Changes in the neural activity were actually involved in malignant atrial remodelling. This finding suggested suggests that the neuronal activity affected the intracellular signal transduction pathways [22], participated in control of the formation of ion flow involved in action potential, and made clear changes in the MAP-, ERP-, and AF-induced rates. This experiment further confirmed that the participation of VNS initiated the electrical remodelling "overlay" phenomenon and exacerbated the changes in the atrial electrophysiological indicators. Moreover, the atrium and pulmonary vein matrix were more to AF. Combined with the current portion of idiopathic AF in clinical studies, patients exhibited arrhythmia, usually accompanied by sinus bradycardia, often when they are at rest or at night. In addition, the patients were insensitive to antiarrhythmic drugs. This condition was considered as high vagal tension. AF aggravated with time when no intervention was administered and will most likely worsen and become persistent AF. These patients were more suitable for treatment involving the blocking of neurological effects, such as GP ablation.

This study confirmed that ICANS and ECANS are dependent on each other and are mutually independent; each system can regulate the activity of another system via the afferent and efferent nerves. ICANS mainly included GP and numerous independent GP-constituted local atrial epicardial or neural networks. However, GP is not only a relay station for nerve signals, but also an integrated signalling or signalling regulation system that can independently regulate the physiological function of the heart. Therefore, GP is known as the "integration center" of neural 
networks [23-25]. Numerous studies have shown that stimulating the nerve plexus can lead to focal AF-induced electrical activity (similar to the activity observed in clinical patients) in the pulmonary vein or atrial junction [26]. The destruction ablation of the GP network also showed that AF induction was significantly reduced after GP ablation, confirming that the exogenous neural input signal was achieved via GP, which is an important gating system. The results are consistent with those of earlier experiments. In addition, AF induction did not completely disappear, especially in atrium, and no obvious pulmonary vein was observed. However, whether the part of exogenous nerve could control the atria by bypassing GP remains unclear. The nerve input of pulmonary veins was blocked because of nerve plexus ablation. However, the direct controlling effects of the atrial myocardium on the endogenous nerve still need to be investigated. GP was close to the pulmonary vein, and the control of GP was stronger than that of the atrium. Nevertheless, this finding requires further experimental demonstration.

Overall, GP ablation significantly eliminated AF in the 2 groups. This finding confirms that GP ablation plays a role in the reversal and prevention of atrial electrical remodelling, regardless of whether electrical remodelling was induced by rapid pacing or by atrial exogenous vagus nerve stimulation because of the undermining ICANS. Therefore, GP is actually involved in various types of AF.

\section{Clinical correlation}

In clinical studies, patients with enhanced vagus nerve tension were prone to $\mathrm{AF}$ and needed maintenance to prevent $\mathrm{AF}$

\section{References:}

1. Nattel S, Harada M: Atrial Remodeling and Atrial Fibrillation: Recent Advances and Translational Perspectives. J Am Coll Cardiol, 2014; 63(22): 2335-45

2. Yuan BX, Ardell JL, Hopkins DA et al: Gross and microscopicanatomy of the canine intrinsic cardiac nervous system. Anat Rec, 1994; 239(1): 75-87

3. Pauza DH, Skripka V, Pauziene $\mathrm{N}$ et al: Morphology, distribution, and variability of the epicardiac neural ganglionated subplexuses in the human heart. Anat Rec, 2000; 259(4): 353-82

4. Beaumont E, Salavatian S, Southerland EM et al: Network interactions within the canine intrinsic cardiac nervous system: implications for reflex control of regional cardiac function. J Physiol, 2013; 591(Pt 18): 4515-33

5. Armour JA: Potential clinical relevance of the "little brain" on the mammalian heart. Exp Physiol, 2008; 93(2): 165-76

6. He B, Scherlag BJ, Nakagawa $\mathrm{H}$ et al: The intrinsic autonomic nervous system in atrial fibrillation: a review. ISRN Cardiol, 2012; 2012: 490674

7. Wickramasinghe SR, Patel VV: Local innervation and atrial fibrillation. Circulation, 2013; 128(14): 1566-75

8. Tan AY, Verrier RL: The role of the autonomic nervous system in cardiac arrhythmias. Handb Clin Neurol, 2013; 117: 135-45

9. Linz D, Ukena C, Mahfoud F et al: Atrial autonomic innervation: a target for interventional antiarrhythmic therapy? J Am Coll Cardiol, 2014; 63(3): 215-24

10. Lu ZB, Scherlag BJ, Lin JX et al: Atrial fibrillation begets atrial fibrillation: Autonomic mechanism for atrial electrical remodeling induced by shortterm rapid atrial pacing. Circ Arrhythm Electrophysiol, 2008; 1(3): 184-92 recurrence or treatment termination. This approach may be achieved by radiofrequency GP ablation.

\section{Limitations}

Sodium pentobarbital was the main anaesthesia drug used in the experiment. This drug enhanced the sympathetic activity, inhibited the sympathomimetic effect of the vagus nerve, and to some extent influenced the results. The experiments were performed in anaesthetized dogs in normal resting state, which lacks the assessment results possible in the environmental state. Moreover, GP+LOM ablation did not guarantee complete blocking of the effect of vagus nerve stimulation. Our results suggest that there are other ways to directly control the atrial nerves. However, the method of bypassing GP needs to be investigated. Therefore, a deceased vagus nerve could not guarantee complete ablation. The experiments also lacked data on nerve discharge. The mechanisms of the occurrence and persistence of AF involved in neural activities could be better explained if the GP discharge was recorded.

\section{Conclusions}

Vagus nerve stimulation "overlay" deteriorated electrical remodelling, and "AF begets AF" was blocked because of epicardial neural network ablation.

\section{Declaration of conflict of interest}

None.

11. Kottkamp H: Human atrial fibrillation substrate: towards a specific fibrotic atrial cardiomyopathy. Eur Heart J, 2013; 34(35): 2731-38

12. Bode F, Kilborn $M$, Karasik $P$ et al: The repolarization-excitability relationship in the human right atrium is unaffected by cycle length, recording siteand prior arrhythmias. J Am Coll Cardiol; 2001; 37(3): 920-25

13. Groves DA, Brown VJ: Vagal nerve stimulation: A review of its applications and potential mechanisms that mediate its clinical effects. Neurosci Biobehav Rev, 2005; 29: 493-500

14. Zhou J, Scherlag BJ, Edwards J et al: Gradients of atrial refractoriness and inducibility of atrial fibrillation due to stimulation of ganglionated plexi. J Cardiovasc Electrophysiol, 2007; 18: 83-90

15. Morillo CA, Klein GJ, Jones DL et al: Chronic rapid atrial pacing. Structural, functional, and electrophysiological characteristics of a new model of sus tained atrial fibrillation. Circulation, 1995; 91(5): 1588-95

16. Wijffels MCEF, Kirchhoff CJHJ, Dorland R et al: Atrial fibrillation begets atrial fibrillation: a study in awake chronically instrumented goats. Circulation, 1995; 92: 1954-68

17. Wijffels MC, Kirchhoff CJ, Dorland R et al: Electrical remodeling due to atrial fibrillation in chronically instrumented conscious goats: roles of neurohumoral changes, ischemia, atrial stretch and high rate of electrical activation. Circulation, 1997; 96: 3710-20

18. Nattel S, Li D, Yue L: Basic mechanisms of atrial fibrillation - very new in sights into very old ideas. Annu Rev Physiol, 2000; 62: 51-77 
19. Voigt N, Heijman J, Wang $Q$ et al: Cellular and molecular mechanisms of atrial arrhythmogenesis in patients with paroxysmal atrial fibrillation. Circulation, 2014; 129(2): 145-56

20. Oh S: Disease modification by autonomic nerve stimulation. Heart Rhythm, 2012; 9(5): 810-11

21. Ng J, Passman RS, Arora R et al: Paradoxical change in atrial fibrillation dominant frequencies with baroreflex-mediated parasympathetic stimulation with phenylephrine infusion. J Cardiovasc Electrophysiol, 2012; 23(10): 1045-50

22. StavrakisS, Scherlag BJ, Fan Y et al: Inhibition of atrial fibrillation by lowlevel vagus nerve stimulation: the role of the nitricoxide signaling pathway. J Interv Card Electrophysiol, 2013; 36(3): 199-208

23. Lo LW, Scherlag BJ, Chang HY et al: Paradoxical long-term proarrhythmic effects after ablating the "head station" ganglionated plexi of the vagal innervation to the heart. Heart Rhythm, 2013; 10(5): 751-57
24. Lo LW, Chiou CW, Lin YJ et al: Neural mechanism of atrial fibrillation: insight from global high density frequency mapping. J Cardiovasc Electrophysiol, 2011; 22(9): 1049-56

25. Po SS, Nakagawa H, Jackman WM: Localization of left atrial ganglionated plexi in patients with atrial fibrillation.J Cardiovasc Electrophysiol, 2009; 20(10): 1186-89

26. Scherlag BJ, Yamanashi W, Patel U et al: Autonomically induced conversion of pulmonary vein focal firing into atrial fibrillation. J Am Coll Cardiol, 2005; 45: 1878-86

27. Schauerte PN, Scherlag BJ, Pitha J et al: Catheter ablation of cardiac autonomic nerves for prevention ofvagal atrial fibrillation. Circulation, 2000 ; 102: $2774-80$

28. Lu Z, Scherlag BJ, Lin J et al: Autonomic mechanism for initiation of rapid firing from atria and pulmonary veins: evidence by ablation of ganglionated plexi. Cardiovasc Res, 2009; 84(2): 245-52 